\title{
Advances in the MQDT approach of electron/molecular cation reactive collisions: High precision extensive calculations for applications
}

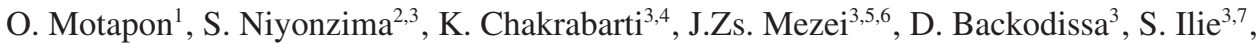 \\ M.D. Epee Epee ${ }^{1}$, B. Peres ${ }^{3,8}$, M. Lanza ${ }^{3}$, T. Tchakoua ${ }^{1}$, N. Pop ${ }^{7}$, F. Argoubi ${ }^{9}$, M. Telmini ${ }^{9}$, \\ O. Dulieu ${ }^{5}$, A. Bultel $^{8}$, J. Robert ${ }^{5}, \AA ̊$. Larson $^{10}$, A.E. Orel ${ }^{11}$ and I.F. Schneider ${ }^{3,5, a}$
}

${ }^{1}$ LPF, UFD/MIAPF, University of Douala, PO Box, 24157 Douala, Cameroon

${ }^{2}$ Département de Physique, Faculté des Sciences, Université du Burundi, BP. 2700, Bujumbura, Burundi

${ }^{3}$ LOMC, CNRS-UMR-6294, Université du Havre, 76058 Le Havre, France

${ }^{4}$ Dept. of Mathematics, Scottish Church College, 1 \& 3 Urquhart Sq., Kolkata 700 006, India

${ }^{5}$ LAC, CNRS-UPR-3321, Univ. Paris-Sud and Ecole Normale Supérieure de Cachan, 91405 Orsay, France

${ }^{6}$ Institute of Nuclear Research of the Hungarian Academy of Sciences, PO Box 51, Debrecen 4001, Hungary

${ }^{7}$ Department of Physical Foundations of Engineering, Politehnica University of Timisoara, Bv. Vasile Parvan No. 2, 300223 Timisoara, Romania

${ }^{8}$ CORIA, UMR CNRS 6614, Université de Rouen, Site Universitaire du Madrillet, Avenue de l'Université, 76801 Saint-Etienne du Rouvray Cedex, France

${ }^{9}$ LSAMA, University of Tunis El Manar, Tunis, Tunisia

${ }^{10}$ Department of Physics, Stockholm University, AlbaNova University Center, 10691 Stockholm, Sweden

${ }^{11}$ Department of Chemical Engineering and Materials Science, University of California, Davis, California 95616, USA

\begin{abstract}
Recent advances in the stepwise multichannel quantum defect theory approach of electron/molecular cation reactive collisions have been applied to perform computations of cross sections and rate coefficients for dissociative recombination and electron-impact rovibrational transitions of $\mathrm{H}_{2}^{+}, \mathrm{BeH}^{+}$and their deuterated isotopomers. At very low energy, rovibronic interactions play a significant role in the dynamics, whereas at high energy, the dissociative excitation strongly competes with all other reactive processes.
\end{abstract}

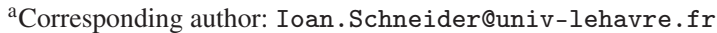

This is an Open Access article distributed under the terms of the Creative Commons Attribution License 4.0, which permits unrestricted use, distribution, and reproduction in any medium, provided the original work is properly cited. 


\section{Introduction}

The collision of an electron of energy $\varepsilon$ with a molecular cation initially in its ground electronic state on its ro-vibrational level $\left(N_{i}^{+}, v_{i}^{+}\right)$results in the formation of various superexcited states, decaying eventually by autoionization and predissociation to different products:

$$
A B^{+}\left(N_{i}^{+}, v_{i}^{+}\right)+e^{-}(\varepsilon) \rightarrow A B^{*}, A B^{*(c)}, A B^{* *}, A B^{* *(c)} \rightarrow\left\{\begin{array}{l}
{[A+B]_{(\epsilon)}} \\
A B^{+}\left(N_{f}^{+}, v_{f}^{+}\right)+e^{-}\left(\varepsilon_{f}\right) \\
A+B_{(\epsilon)}^{+}+e^{-}\left(\varepsilon_{f}\right)
\end{array}\right.
$$

According to the type of these products, different processes take place: dissociative recombination (DR 1(a)), elastic, inelastic or superelastic collisions (EC, IC, SEC (1b)), corresponding to the final energy of the electron $\varepsilon_{f}$ equal, smaller or larger than $\varepsilon$ respectively, and, if $\varepsilon$ exceeds the dissociation energy of the target cation, electron impact ion dissociation, i.e. dissociative excitation (DE (1c))[1-4]. Here $\mathrm{AB}^{*}$ stands for states bound from both electronic and vibrational point of view, $\mathrm{AB}^{*(c)}$ for states from the mono-electronic continuum (superscript (c)), $\mathrm{AB}^{* *}$ for states bound from the electronic point of view but dissociative (i.e. from the vibrational continuum), $\mathrm{AB}^{* *(c)}$ for states from the mono-electronic continuum and dissociative, and $\epsilon$ for the relative kinetic energy release of the heavy products. Eq. (1) are appropriate for diatomic systems, but can be generalized for polyatomic ones.

All the above processes drive the particle densities in fusion plasmas close to the walls and in the divertor region [5-8], in various media of astrophysical interest [9-12], in the hypersonic entry plasmas [13], and in many other environments of fundamental or technological relevance.

The present paper is, first of all, a brief review of the recent developments in our theoretical approach - concerning namely rotational transitions at low collision energies and the dissociative autoionization (dissociative excitation) at high collision energies. Secondly, it illustrates some of our new results on $\mathrm{H}_{2}^{+} / \mathrm{H}_{2}$ systems and $\mathrm{BeH}^{+} / \mathrm{BeH}$ systems (including their isotopomers), devoted to the kinetic modeling of gaseous ionized media in the early Universe, interstellar molecular clouds and edge fusion plasmas.

Sections 2 reviews the theory. Section 3 presents some of our new results and their comparison to previous computations. Section 4 contains the conclusions and the perspectives.

\section{The stepwise MQDT-type approach for electron/molecular cation reactive collisions}

The dynamics of the infinite series of $\mathrm{AB}^{*}$ states, often called "Rydberg resonances", appearing in (1), is elegantly and efficiently modeled by a method [14-18] based on the multichannel quantum defect theory (MQDT) [19-22]. The processes (1) result from the coupling between ionization and dissociation channels, i.e. groups of states characterized by a common set of quantum numbers and by the same fragmentation threshold (either for ionization, or for dissociation), having the energy below or above this threshold. More specifically, within a quasi-diabatic representation [15, 16, 23], an ionization channel is built starting from the ground electronic state of the ion in one of its ro-vibrational levels $N^{+} v^{+}$, and is completed by gathering all the mono-electronic states of a given orbital quantum number $l$, describing an "optical" electron. These mono-electronic states describe, with respect to the $N^{+} v^{+}$ threshold, either a "free" electron - in which case the total state $\mathrm{AB}^{*(c)}$ corresponds to (auto)ionization or to a bound one - in which case the total state $\mathrm{AB}^{*}$ corresponds to a temporary capture into a Rydberg state. Meanwhile, a dissociation channel relies on an electronically bound state $\mathrm{AB}^{* *}$ whose potential energy in the asymptotic limit is situated below the total energy of the system.

Accordingly, the ionization channels gather together $\mathrm{AB}^{*}$ and $\mathrm{AB}^{*(c)}$ states, and the dissociation channels correspond to $\mathrm{AB}^{* *}$ states. As for those of $\mathrm{AB}^{* *(c)}$ type, they can be organized either into dissociation, or into ionization channels, but the latter option has been preferred so far. Given the 
total energy of the molecular system, a channel is open if this energy is higher than the energy of its threshold, and closed in the opposite case. In the modeling of the reactions (1), relying exclusively on the open channels means accounting for the direct mechanism only. The inclusion of the closed channels - including $\mathrm{AB}^{*}$ states - allows for the indirect mechanism, which interferes with the direct one resulting in the total process.

Our MQDT approach starts with the building of the interaction matrix $\mathcal{V}$, performed in the "A-region" [24], where the Born-Oppenheimer context is appropriate for the description of the collision system. The good quantum numbers in this region are $N, M$, and $\Lambda$, associated respectively to the total angular momentum and its projections on the z-axis of the laboratory-fixed and of the moleculefixed frames. In the A-region, the states belonging to an ionization channel may be modeled reasonably well with respect to hydrogenic states in terms of the quantum defect $\mu_{l}^{\Lambda}$, which is dependent on the internuclear distance $R$, but assumed to be independent of energy. An ionization channel is coupled to a dissociation one, labeled $d_{j}$, on electronic level first, through an $R$-dependent scaled "Rydbergvalence" interaction term, $V_{d_{j}, l}^{(e) \Lambda}$, which is assumed to be independent of the energy of the electronic states pertaining to the ionization channel. Integrating this coupling over the internuclear distance gives elements of the interaction matrix $\mathcal{V}$ :

$$
\mathcal{V}_{d_{j}, l N^{+} v^{+}}^{N M \Lambda}(E, E)=\left\langle\chi_{N d_{j}}^{\Lambda}\left|V_{d_{j}, l}^{(e) \Lambda}\right| \chi_{N^{+}, v^{+}}^{\Lambda}\right\rangle .
$$

The dependence on the total energy $E$ of the couplings are carried by $\chi_{d_{j}}^{\Lambda}$, the nuclear wave-functions corresponding to a dissociative state and by $\chi_{N^{+}, v^{+}}^{\Lambda}$, the wave-functions corresponding to an ionization channel, respectively. This procedure applies in each $\Lambda$-subspace, and results in a block-diagonal global interaction matrix. Starting from the interaction matrix $\mathcal{V}$ and from the zero-order Hamiltonian $\boldsymbol{H}_{\mathbf{0}}$, we build the reaction K-matrix, which satisfies the Lippmann-Schwinger equation [25]:

$$
\mathcal{K}=\mathcal{V}+\mathcal{V} \frac{1}{E-\boldsymbol{H}_{\mathbf{0}}} \mathcal{K}
$$

In order to express the result of the short-range interaction in terms of phase-shifts, we perform a unitary transformation of our initial basis into a new one, corresponding to eigenchannels, via the diagonalization of the reaction matrix $\mathcal{K}$ :

$$
\mathcal{K} \mathbf{U}=-\frac{1}{\pi} \tan (\eta) \mathbf{U}
$$

In the external "B-region" [24] the Born-Oppenheimer model is no longer valid for the neutral molecule, and a frame transformation [26-28] is performed, via the projection coefficients:

$$
\begin{gathered}
\mathcal{C}_{l N^{+} v^{+}, \Lambda \alpha}=\left(\frac{2 N^{+}+1}{2 N+1}\right)^{1 / 2}\left\langle l\left(\Lambda-\Lambda^{+}\right) N^{+} \Lambda^{+} \mid l N^{+} N \Lambda\right\rangle \\
\times \frac{1+\tau^{+} \tau(-1)^{N-l-N^{+}}}{\left[2\left(2-\delta_{\Lambda^{+}, 0}\right)\left(1+\delta_{\Lambda^{+}, 0} \delta_{\Lambda, 0}\right)\right]^{1 / 2}} \times \sum_{v} U_{l v, \alpha}^{\Lambda}\left\langle\chi_{N^{+} v^{+}}^{\Lambda^{+}}\left|\cos \left(\pi \mu_{l}^{\Lambda}(R)+\eta_{\alpha}^{\Lambda}\right)\right| \chi_{N v}^{\Lambda}\right\rangle, \\
\mathcal{C}_{d_{j}, \Lambda \alpha}=U_{d_{j} \alpha}^{\Lambda} \cos \eta_{\alpha}^{\Lambda},
\end{gathered}
$$

which can be organized in a matrix $\mathcal{C}$. The other projection coefficients organized in a matrix $\mathcal{S}$, are obtained as $\mathcal{S}_{l N^{+} v^{+}, \Lambda \alpha}$ and $\mathcal{S}_{d_{j}, \Lambda \alpha}$ by replacing cosine with sine in Eqs. (4) and (5). In these equations, the quantities $\tau^{+}$and $\tau$ are related to the reflection symmetry of the ion and neutral wave function respectively, and take the values $+1 /-1$ for symmetric/antisymmetric states respectively. 
Matrices $\mathcal{C}$ and $\mathcal{S}$ are the building blocks of the generalized scattering matrix $\boldsymbol{X}$, involving all channels, open ("o") and closed ("c"), and organized in 4 sub-matrices:

$$
X=\frac{\mathcal{C}+i \mathcal{S}}{\mathcal{C}-i \mathcal{S}}, \quad X=\left(\begin{array}{ll}
X_{o o} & X_{o c} \\
X_{c o} & X_{c c}
\end{array}\right)
$$

Imposing boundary conditions leads to the physical scattering matrix [19]:

$$
\boldsymbol{S}=\boldsymbol{X}_{o o}-\boldsymbol{X}_{o c} \frac{1}{\left.\boldsymbol{X}_{c c}-\exp (-\mathrm{i}] 2 \mathrm{p} \boldsymbol{v}\right)} \boldsymbol{X}_{c o},
$$

where the diagonal matrix $\boldsymbol{v}$ is formed with the effective quantum numbers $v_{N^{+} v^{+}}=\left[2\left(E_{N^{+} v^{+}}-E\right)\right]^{-1 / 2}$ (in atomic units) associated with each vibrational threshold $E_{N^{+} v^{+}}$of the ion situated above the current energy $E$ (and consequently labeling a closed channel).

For a molecular ion initially in the level $N_{i}^{+} v_{i}^{+}$recombining with an electron of kinetic (collision) energy $\varepsilon$, the cross section of capture into all the dissociative states $d_{j}$ of the same symmetry is given by

$$
\sigma_{\mathrm{diss} \leftarrow N_{i}^{+} v_{i}^{+}}^{N, \mathrm{sym}}=\frac{\pi}{4 \varepsilon} \frac{2 N+1}{2 N_{i}^{+}+1} \rho^{\mathrm{sym}} \sum_{l, \Lambda, j}\left|S_{d_{j}, l N_{i}^{+} v_{i}^{+}}^{N \Lambda}\right|^{2} .
$$

On the other hand, the cross section for a ro-vibrational transition to the final level $N_{f}^{+} v_{f}^{+}$, giving reactive elastic scattering or (de-)excitation, writes:

$$
\begin{aligned}
\sigma_{N_{f}^{+} v_{f}^{+} \leftarrow N_{i}^{+} v_{i}^{+}}^{N, \text { sm }}= & \frac{\pi}{4 \varepsilon} \frac{2 N+1}{2 N_{i}^{+}+1} \rho^{\mathrm{sym}} \\
& \times \sum_{l, l^{\prime}, \Lambda, j}\left|S_{N_{f}^{+} v_{f}^{+} l^{\prime}, N_{i}^{+} v_{i}^{+} l}-\delta_{N_{f}^{+} N_{i}^{+}} \delta_{v_{f}^{+} v_{i}^{+}} \delta_{l^{\prime} l}\right|^{2} .
\end{aligned}
$$

Here $\rho^{\text {sym }}$ is the ratio between the multiplicities of the neutral and the target ion. After performing the MQDT calculations for all accessible total rotational quantum numbers $N$ and for all the relevant symmetries, the global cross section for dissociative recombination or ro-vibrational (de-)excitation as a function of the electron collision energy $\varepsilon$ reads as:

$$
\sigma_{\text {diss } \leftarrow N_{i}^{+} v_{i}^{+}}=\sum_{\text {sym }, N} \sigma_{\text {diss } \leftarrow N_{i}^{+} v_{i}^{+}}^{N, \text { sym }}, \quad \sigma_{N_{f}^{+} v_{f}^{+} \leftarrow N_{i}^{+} v_{i}^{+}}=\sum_{\text {sym }, N} \sigma_{N_{f}^{+} v_{f}^{+} \leftarrow N_{i}^{+} v_{i}^{+}}^{N,}
$$

When rotational excitation and rotational couplings are neglected - the so-called "non-rotational case" - the formalism becomes much simpler. Moreover, one has to perform separate calculations within each symmetry block $\Lambda$ and eventually sum over this quantum number the resulting cross sections. For a given $\Lambda$, the previous formulas become:

$$
\begin{gathered}
\mathcal{V}_{d_{j}, l v^{+}}^{\Lambda}(E, E)=\left\langle\chi_{d_{j}}^{\Lambda}\left|V_{d_{j}, l}^{(e) \Lambda}\right| \chi_{v^{+}}\right\rangle, \\
\mathcal{C}_{l v^{+}, \Lambda \alpha}=\sum_{v} U_{l v, \alpha}^{\Lambda}\left\langle\chi_{v^{+}}(R)\left|\cos \left(\pi \mu_{l}^{\Lambda}(R)+\eta_{\alpha}^{\Lambda}\right)\right| \chi_{v}(R)\right\rangle, \quad \mathcal{C}_{d, \Lambda \alpha}=U_{d \alpha}^{\Lambda} \cos \eta_{\alpha}^{\Lambda}, \\
\sigma_{\text {diss } \longleftarrow v_{i}^{+}}^{\mathrm{sym}, \Lambda}=\frac{\pi}{4 \varepsilon} \rho^{\mathrm{sym}, \Lambda} \sum_{l, j}\left|S_{d_{j}, l v_{i}^{+}}^{\Lambda}\right|^{2}, \quad \sigma_{\mathrm{diss} \longleftarrow v_{i}^{+}}=\sum_{\Lambda, \text { sym }} \sigma_{\text {diss } \longleftarrow v_{i}^{+}}^{\mathrm{sym}, \Lambda}
\end{gathered}
$$




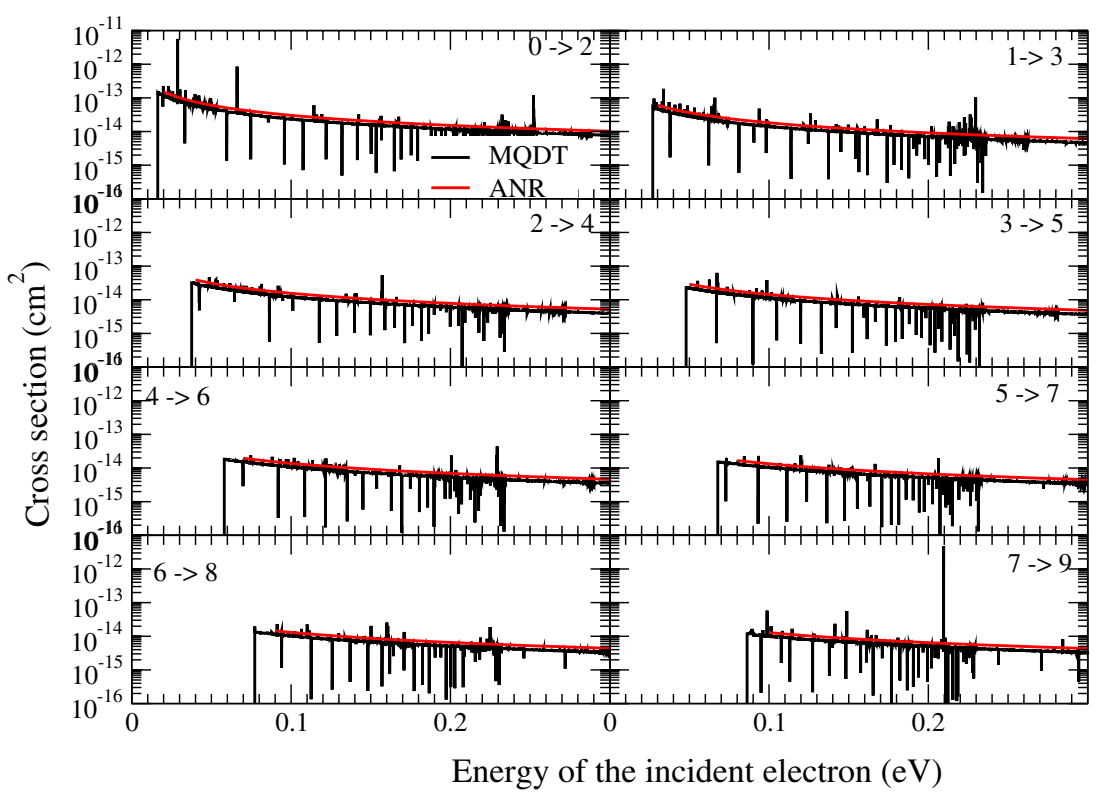

Figure 1. Cross sections for rotational excitation $N_{i}^{+}-2 \rightarrow N_{i}^{+}$, with $N_{i}^{+}=2$ to 9 , of vibrationally relaxed $\mathrm{HD}^{+}\left(X^{2} \Sigma_{g}^{+}\right)$. Black curves: MQDT computations; red curves: ANR approximation based computations [30].

The cross section for vibrational transition - reactive elastic scattering or vibrational (de)-excitation - is expressed as:

$$
\begin{gathered}
\sigma_{v_{f}^{+} \leftarrow v_{i}^{+}}^{\mathrm{sym}, \Lambda}=\frac{\pi}{4 \varepsilon} \rho^{\mathrm{sym}, \Lambda} \sum_{l, l^{\prime}}\left|S_{l^{\prime} v_{f}^{+}, l v_{i}^{+}}-\delta_{l^{\prime} l} \delta_{v_{f}^{+} v_{i}^{+}}\right|^{2}, \\
\sigma_{v_{f}^{+} \leftarrow v_{i}^{+}}=\sum_{\Lambda, \mathrm{sym}} \sigma_{v_{f}^{+} \leftarrow v_{i}^{+}}^{\mathrm{sym}, \Lambda}
\end{gathered}
$$

At energies higher than the dissociation threshold of the ion, we have to take into account the autoionization into states from the continuum part of the vibrational spectrum, i.e., dissociative excitation (DE, (1c). These states, representing a free electron in the field of a dissociating ion, can be organized either into dissociation channels or into ionization ones, but the latter option has been preferred so far. If the lowest two electronic states of the ion target - whose potential energy curves (PEC) will be labelled core 1 and core 2 - have the same dissociation limit, the states responsible for the $\mathrm{DE}$ are represented by an electron in the ionization continuum associated to a vibrationally dissociative state of either of these cores. We have discretized these vibrational continua by providing a wall of $15 \mathrm{eV}$ height at $R=25$ a.u. This results in about 400 further ionization channels associated to core 1 and core 2 , responsible respectively for what we call dissociative excitation of the first kind (DE1) and of the second kind (DE2).

Consequently, the coupling between a given dissociation channel $d_{j}$ and an ionization one $v^{+}$, built on core 1 (Eq. (11)) is extended to the continuum part of the vibrational spectrum. Furthermore, every channel $v^{+}$is coupled to the ionization channels built on core 2, since the latter rely on electronic states with similar configuration as the $d_{j}$ states. One should also notice that since the temporary capture into bound Rydberg states $A B^{*}$ is excluded above the dissociation limit of the ion PEC, the collision process is exclusively driven by the direct mechanism. 


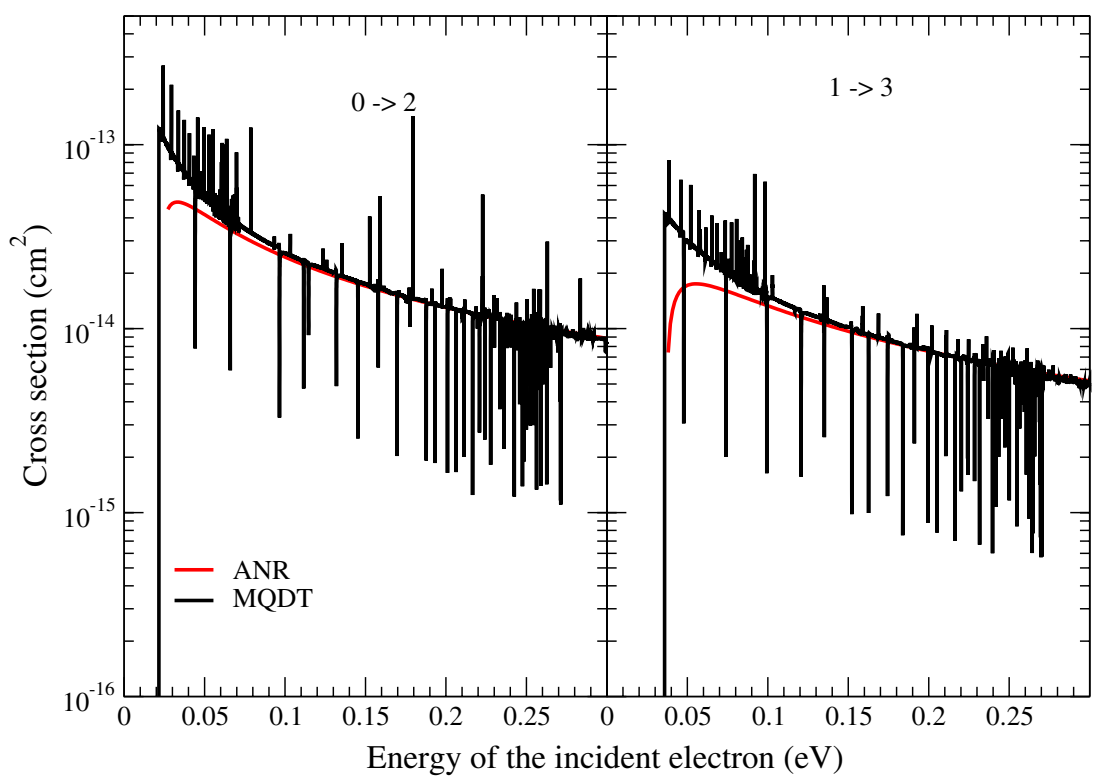

Figure 2. Cross sections for the rotational excitation $0 \rightarrow 2$ and $1 \rightarrow 3$ of vibrationally relaxed $\mathrm{H}_{2}^{+}$. The MQDT results (black lines) are compared with those from Faure and Tennyson [31] using the ANR/R-matrix method (red lines).

Further details on the way that formulas $(6,7,12-15)$ generalize to DE and DR competed by DE are given in [29].

\section{New results on $\mathrm{H}_{2}^{+}, \mathrm{BeH}^{+}$and isotopomers}

\subsection{Very low energy: Ro-vibronic interactions}

After having intensively studied the dissociative recombination and the vibrational transitions in $\mathrm{H}_{2}^{+}$ and $\mathrm{HD}^{+}[18,32]$, we have recently focused on the electron-impact rotational transitions of these ions at very low collision energies [30].

Figure 1 displays our results for vibrationally relaxed $\mathrm{HD}^{+}\left(X^{2} \Sigma_{g}^{+}\right)$, compared with those obtained by the adiabatic nuclei rotation (ANR) approximation. One can notice that the cross sections are dominated by resonance structures due to the indirect process that is the temporary capture into the numerous Rydberg states of the neutral system (HD*).

Cross sections for rotational excitation of $\mathrm{H}_{2}^{+}$are represented in Fig. 2 for the transitions $0 \rightarrow 2$ and $1 \rightarrow 3$, in comparison with those obtained by Faure and Tennyson [31] using the ANR approximation, with which they agree quite satisfactorily both in shape and magnitude.

Isotropic rate coefficients for the excitation of the lowest two rotational levels of $\mathrm{H}_{2}^{+}$and $\mathrm{HD}^{+}$, obtained from the convolution of cross sections with a Maxwell-Boltzmann velocity distribution function, are represented in Fig. 3, which illustrates the significant magnitude of the isotopic effect.

\subsection{Energy below the ion dissociation threshold: Vibronic interactions}

Using the molecular data on superexcited $\mathrm{BeH}$ states available from our previous studies [33, 34] computations of cross sections and rate coefficients for all the vibrational states of the target have been 


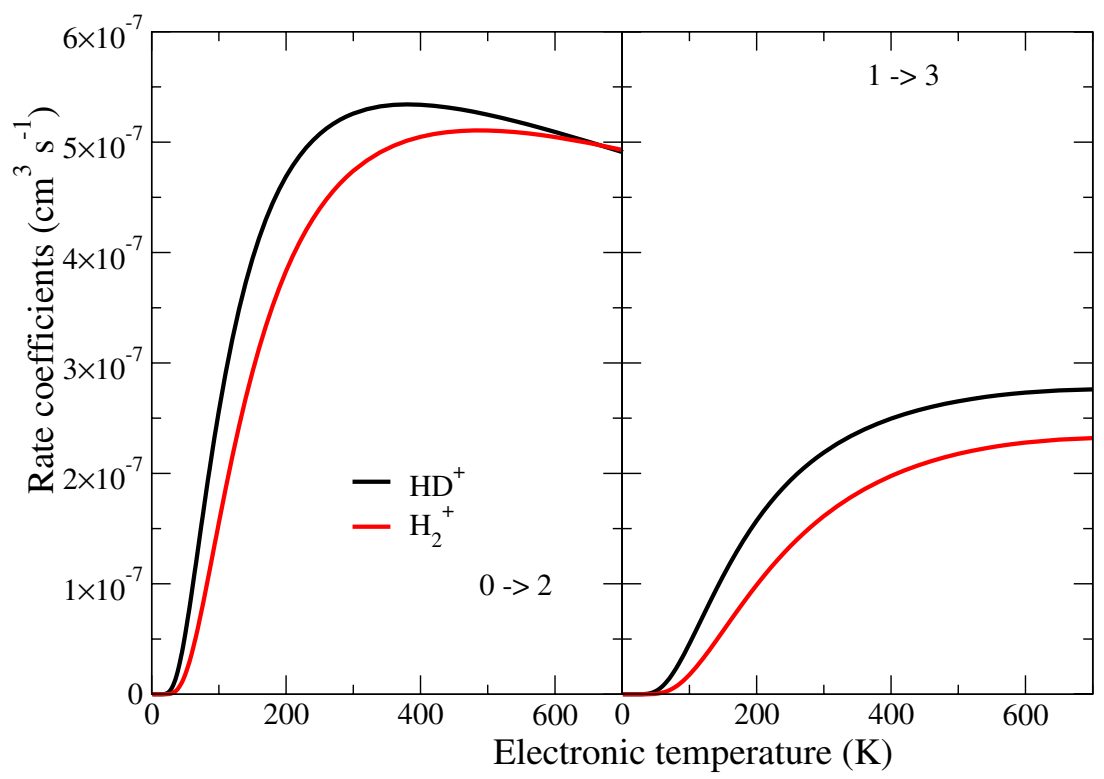

Figure 3. Isotopic effects in rotational exciation of $\mathrm{H}_{2}^{+}$: rate coefficients for $0 \rightarrow 2$ and $1 \rightarrow 3$ transitions in vibrationally relaxed $\mathrm{H}_{2}^{+}$and $\mathrm{HD}^{+}$.

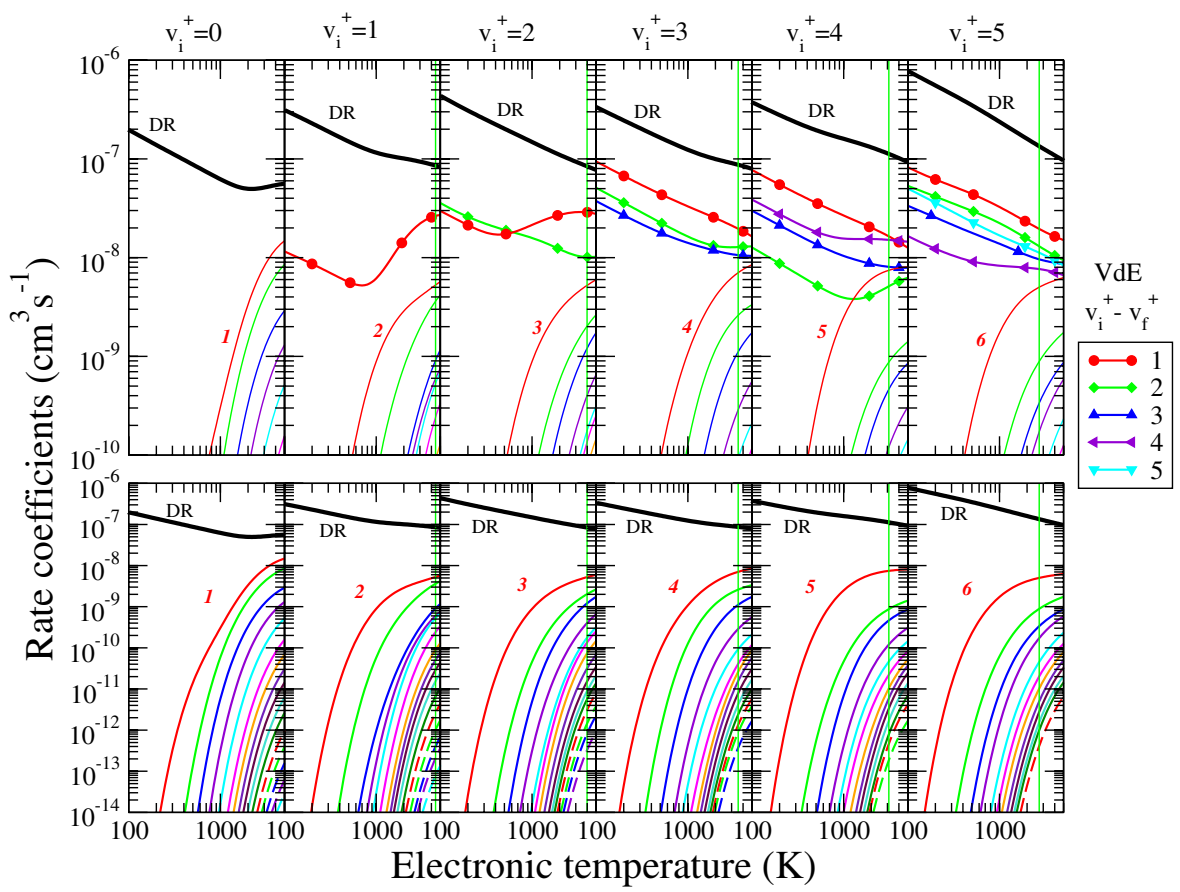

Figure 4. Upper panel: DR and state-to-state VE and VdE rate coefficients of $\mathrm{BeH}^{+}$in its ground electronic state, $\mathrm{v}_{i}^{+}$standing for the vibrational quantum number of the target ion. Curves of same color show the rate coefficients for the vibrational (de-)excitations corresponding to the same $|\Delta v|=\left|v_{f}^{+}-v_{i}^{+}\right|, v_{f}>v_{i}$ for the $\mathrm{VE}$ and $v_{f}<v_{i}$ for the VdE global rate coefficients, respectively. For VE only the lowest final vibrational quantum number of the ion $\mathrm{BeH}^{+}$is indicated. Lower panel: the same DR and VE rate coefficients, on a larger range of values. 


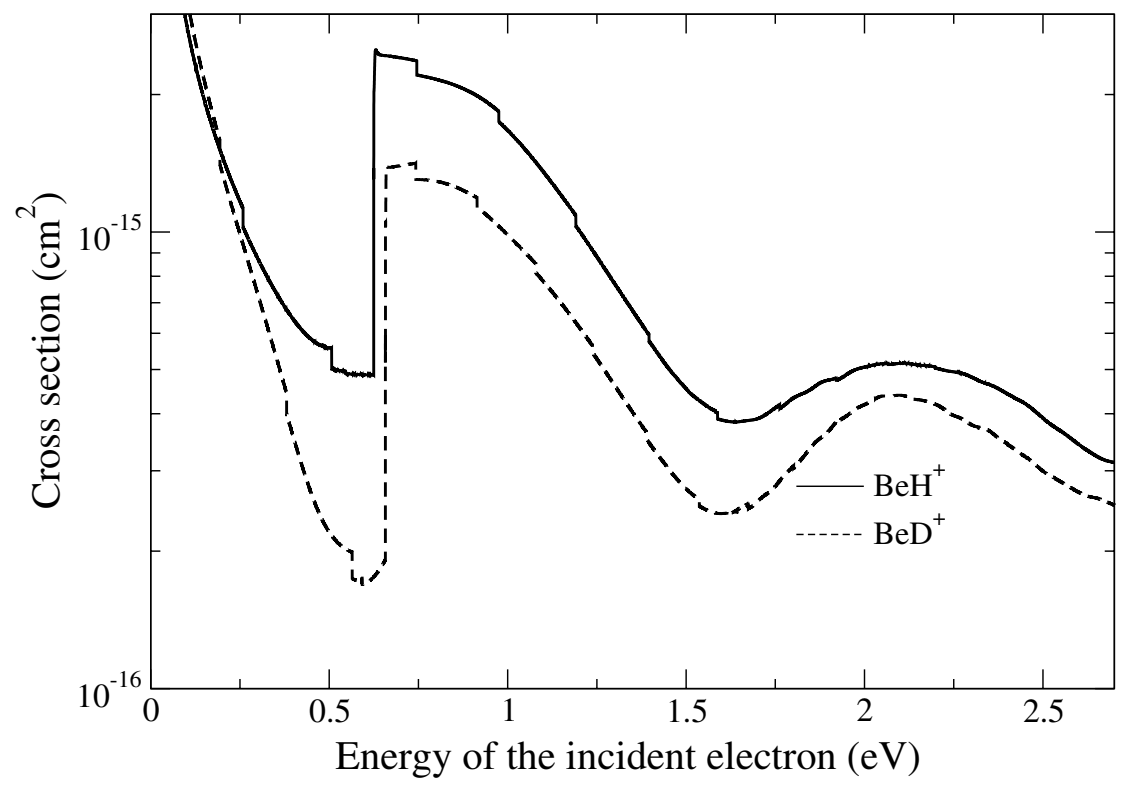

Figure 5. Isotopic effects in low energy $\mathrm{BeH}^{+}$dissociative recombination (direct mechanism).

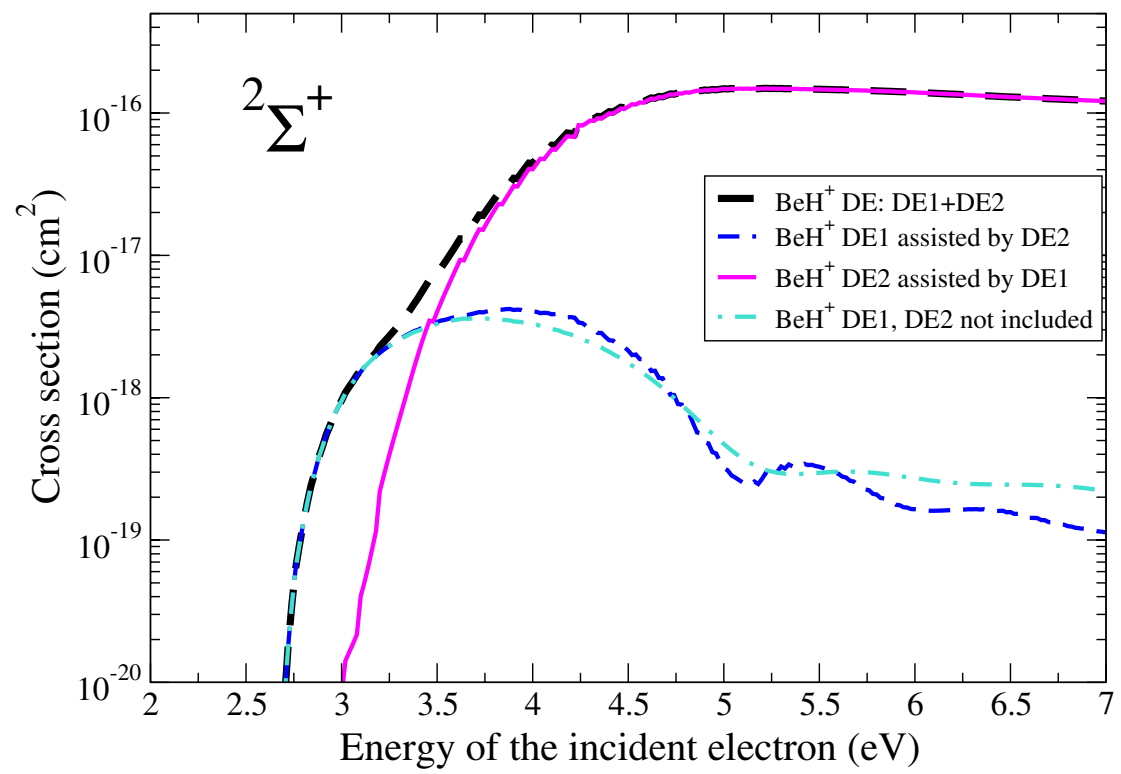

Figure 6. Dissociative excitation of $\mathrm{BeH}^{+}$within the ${ }^{2} \Sigma^{+}$symmetry of $\mathrm{BeH}$. Black dashed curve stands for the $\mathrm{DE}$, the dashed blue/continuous magenta curves give the DE1/DE2 cross sections with simultaneous treatment of DE2/DE1 (DE1 assisted by DE2/DE2 assisted by DE1), while the dashed-dotted cyan curve is the DE1 cross section without DE2.

performed for the electron-impact DR, vibrational excitation (VE) and de-excitation (VdE) of $\mathrm{BeH}^{+}$at low energy (i.e. below the ion dissociation threshold). 
The results concerning the lowest vibrational levels are displayed in Fig. 4. In view of versatile use in kinetics calculations, we have produced generalized Arhenius-type fit formulas, their coefficients being displayed elsewhere [35].

The isotopic effects are illustrated in Fig. 5, where the direct mechanism has been considered exclusively, since as shown in [34], the indirect process is very weak for the electron-impact processes involving this ion. The cross sections for the two isotopomers show the same patterns and thresholds, due to the consecutive opening of different dissociation and ionization channels, while the difference between them is attributed to the different vibrational level densities, due to the different reduced masses.

\subsection{Energy above the ion dissociation threshold: dissociative excitation}

Figure 6 illustrates the evolution of the two DE mechanisms - DE1 and DE2 - with the energy of the incident electron, within one of the relevant symmetries of the $\mathrm{BeH}$ system, ${ }^{2} \Sigma^{+}$. Whereas DE1 is significant in a limited range above the dissociation threshold of the ion and quickly decreases subsequently, DE2 strongly increases over a larger energy range and clearly dominates within a highvalue plateau the DE process.

\section{Conclusion and perspectives}

Using the multichannel quantum defect theory, we have computed cross sections and rate coefficients for electron-impact processes involving $\mathrm{H}_{2}^{+}, \mathrm{BeH}^{+}$, and their deuterated isotopomers.

In the case of $\mathrm{H}_{2}^{+}$, the rotational excitation cross sections and rate coefficients production have to be extended to transitions involving still higher rotational levels, and the dissociative recombination results have to be compared with the latest experimental data.

On the other hand, the study of the contribution of all the relevant symmetries to the dissociative excitation of $\mathrm{BeH}^{+}$has to be completed, and the DE-assisted DR cross sections and rate coefficients to be computed. In order to extend these studies to even higher energies, further dissociative states have to be either computed or modeled, using the scaling laws characterizing the Rydberg series of states.

The authors thank Ch. Jungen for his constant scientific support and fruitful discussions. They acknowledge support from the International Atomic Energy Agency via the Coordinated Research Projects "Light Element Atom, Molecule and Radical Behaviour in the Divertor and Edge Plasma Regions" and "Atomic and Molecular Data for state-Resolved Modelling of Hydrogen and Helium and their isotopes in Fusion" and contract no 16712, from Agence Nationale de la Recherche via the projects "SUMOSTAI" (No. ANR-09-BLAN-020901) and "HYDRIDES" (No. ANR-12-BS05-0011-01), from the IFRAF-Triangle de la Physique via the project "SpecoRyd," and from the Centre National de la Recherche Scientifique via the programs "Physique et Chimie du Milieu Interstellaire" and the PEPS projects "Physique théorique et ses interfaces" TheMS and TPCECAM. They also acknowledge generous financial support from La Région Haute-Normandie via the CPER "THETE" project, and the GRR Electronique, Energie et Matriaux, from the "Fédération de Recherche Energie, Propulsion, Environnement," and from the LabEx EMC ${ }^{3}$, via the project PicoLIBS (No. ANR-10-LABX-09-01). ÅL acknowledges support from the Swedish research council.

\section{References}

[1] M. Larsson and A. E. Orel, Dissociative recombination of molecular ions (Cambridge Univ. Press) (2008)

[2] A. Wolf, L. Lammich and P. Schmelcher (editors), J. Phys. Conf. Series 4, Proceedings of the Sixth International Conference on Dissociative Recombination: Theory, Experiments and Applications, 12-16 July 2004, Mosbach, Germany (2005) 
[3] W. J. van der Zande (editor), J. Phys. Conf. Series 192, Proceedings of the Seventh International Conference on Dissociative Recombination: Theory, Experiments and Applications, 18-23 July 2007, Ameland, The Netherlands (2009)

[4] A. E. Orel and S. L. Guberman (editors), J. Phys. Conf. Series 300, Proceedings of the Seventh International Conference on Dissociative Recombination: Theory, Experiments and Applications, August 16-20 2010, Lake Tahoe, USA (2011)

[5] H. P. Summers, N. R. Badnell, M. G. O’Mullane, A. D. Whiteford, R. Bingham, B. J. Kellett, J. Lang, K. H. Behringer, U. Fantz, K.-D. Zastrow, S. D. Loch, M. S. Pindzola, D. C. Griffin C. P. Ballance, Plasma Physics and Controlled Fusion 44, B323 (2002)

[6] U. Fantz, and P. T. Greenland, Contrib. Plasm. Phys. 42, 694 (2002)

[7] R. E. H. Clark and D. Reiter (Eds.), Nuclear Fusion Research: Understanding Plasma-Surface Interactions, Springer series in Chemical Physics 78 (Springer Berlin Heidelberg) (2005)

[8] R. Celiberto, R. K. Janev and D. Reiter, Plasma Phys. Control. Fusion 54, 035012 (2012)

[9] C. M. Coppola, S. Longo, M. Capitelli, F. Palla, and D. Galli, ApJ. Suppl. Ser. 193, pp. 7 (2011)

[10] P. Gnacinski, A\&A 532, pp. 122 (2011)

[11] J. H. Black, Phil. Trans. R. Soc. A 370, 5130 (2012)

[12] C. D. Gay, N. P. Abel, R. L. Porter, P. C. Stancil, G. J. Ferland, G. Shaw, P. A. M. van Hoof and R. J. R. Williams, ApJ 746, pp. 78 (2012)

[13] A. Bultel, B. G. Chéron, A. Bourdon, O. Motapon and I. F. Schneider, Phys. Plasmas 13, 043502 (2006)

[14] U. Fano, Phys. Rev. 124, 1866 (1961)

[15] N. Bardsley, J. Phys. B 1, 349, 365 (1968)

[16] A. Giusti-Suzor, J. Phys. B: At. Mol. Phys. 13, 3867 (1980)

[17] A. Giusti-Suzor, J. N. Bardsley and C. Derkits, Phys. Rev. A 28, 682 (1983)

[18] O. Motapon, F. O. Waffeu Tamo, X. Urbain and I. F. Schneider, Phys. Rev. A 77, 052711 (2008)

[19] M. J. Seaton, Rept. Prog. Phys. 46, 167 (1983)

[20] C. H. Greene and Ch. Jungen, Adv. At. Mol. Phys. 21, 51 (1985)

[21] Ch. Jungen (editor), Molecular Applications of Quantum Defect Theory, (IoP Publishing, Bristol), (1996)

[22] Ch. Jungen, in Handbook of High resolution Spectroscopy, edited by M. Quack and F. Merkt (Wiley, Chichester/New York) (2011)

[23] V. Sidis and H. Lefèbvre-Brion, J. Phys. B: At. Mol. Phys. 4, 1040 (1971)

[24] Ch. Jungen and O. Atabek, J. Chem. Phys. 66, 5584 (1977)

[25] V. Ngassam, A. Florescu, L. Pichl, I. F. Schneider, O. Motapon and A. Suzor-Weiner, Euro. Phys. J. D 26, 165 (2003)

[26] U. Fano, Phys. Rev. A 2, 353 (1970)

[27] E. S. Chang and U. Fano, Phys. Rev. A 6, 173 (1972)

[28] B. Vâlcu, I. F. Schneider, M. Raoult, C. Strömholm, M. Larsson and A. Suzor-Weiner, Euro. Phys. J. D 1, 71 (1998)

[29] K. Chakrabarti, D. R. Backodissa-Kiminou, N. Pop, J. Zs. Mezei, O. Motapon, F. Lique, O. Dulieu, A. Wolf, and I. F. Schneider, Phys. Rev. A 87, 022702 (2013)

[30] O. Motapon, N. Pop, F. Argoubi, J. Zs Mezei, M. D. Epee Epee, A. Faure, M. Telmini, J. Tennyson, and I. F. Schneider, Phys. Rev. A 90, 012706 (2014)

[31] A. Faure and J. Tennyson, MNRAS 325, 443 (2001)

[32] F. O. Waffeu Tamo, Buhr, H., O. Motapon, S. Altevogt, V. M. Andrianarijaona, M. Grieser, L. Lammich, M. Lestinsky, M. Motsch, I. Nevo, S. Novotny, D. A. Orlov, H. B. Pedersen, D. Schwalm, F. Sprenger, X. Urbain, U. Weigel, A. Wolf, and I. F. Schneider, Phys. Rev. A 84, $022710(2011)$ 
[33] J. B. Roos, M. Larsson, Å. Larson and A. E. Orel, Phys. Rev. A 80, 012501 (2009)

[34] S. Niyonzima, F. Lique, K. Chakrabarti, Å. Larson, A. E. Orel, I. F. Schneider, Phys. Rev. A 87, 022713 (2013)

[35] J. Zs. Mezei, S. Niyonzima, D. Backodissa, N. Pop, F. O. Waffeu Tamo, K. Chakrabarti, O. Motapon, A. Wolf, J. Robert, O. Dulieu, Å. Larson, A. E. Orel, A. Bultel, and I. F. Schneider, J. Phys. Conf. Ser. 576, 012005 (2015) 